\title{
Blow-Up Phenomena for Porous Medium Equation with Nonlinear Flux on the Boundary
}

\author{
Yan $\mathrm{Hu}^{1}{ }^{1}$ Jing $\mathrm{Li}^{2}{ }^{2}$ and Liangwei Wang ${ }^{1}$ \\ ${ }^{1}$ School of Mathematics and Statistics, Chongqing Three Gorges University, Wanzhou 404100, China \\ ${ }^{2}$ College of Science, Minzu University of China, Beijing 100081, China
}

Correspondence should be addressed to Yan Hu; 450729639@qq.com

Received 19 July 2013; Accepted 1 November 2013

Academic Editor: Malgorzata Peszynska

Copyright (c) 2013 Yan Hu et al. This is an open access article distributed under the Creative Commons Attribution License, which permits unrestricted use, distribution, and reproduction in any medium, provided the original work is properly cited.

We investigate the blow-up phenomena for nonnegative solutions of porous medium equation with Neumann boundary conditions. We find that the absorption and the nonlinear flux on the boundary have some competitions in the blow-up phenomena.

\section{Introduction}

In this paper, we are concerned with the blow-up of solutions of porous medium equations with nonlinear flux on the boundary. Consider

$$
\begin{gathered}
u_{t}=\Delta u^{m}-f(u), \quad(x, t) \in \Omega \times\left(0, t^{*}\right), \\
\frac{\partial u^{m}}{\partial v}=g(u), \quad(x, t) \in \partial \Omega \times\left(0, t^{*}\right), \\
u(x, 0)=u_{0}(x), \quad x \in \Omega,
\end{gathered}
$$

where $m>1$, the nonnegative initial value $u_{0}(x) \in$ $C(\Omega) \cap L^{\infty}(\Omega), \Omega$ is a bounded region in $\mathbb{R}^{N}(N \geq 2)$ with the sufficiently smooth boundary $\partial \Omega, v$ is the unit normal vector on $\partial \Omega, t^{*}$ is the blow-up time if blow-up occurs, or else $t^{*}=\infty$.

The blow-up phenomena for the nonnegative solutions of the heat equation with nonlinear sources $(m=1$ and $f(u)=$ $-u^{p}$ in (1)) in the whole space $\mathbb{R}^{N}$ was first found by Fujita in 1966, see [1]. He proved the following results:

(a) if $1<p<1+(2 / N)$, then (1) has no global positive solutions;

(b) if $p>1+(2 / N)$, then there exist global positive solutions.

The critical case $p=1+(2 / N)$ was proved to belong to the blow-up case in 1970's by several authors [2-4]. In
1980, Galaktionov and others [5] considered the nonnegative solutions of (1) (with $m>1$ and $f(u)=-u^{p}$ ) in whole space $\mathbb{R}^{N}$. They found some results similar to those for the heat equation $(m=1)$ as follows

(a) if $1<p<m+(2 / N)$, then (1) has no global solutions;

(b) if $p>m+(2 / N)$, then there exist global positive solutions that decay like $t^{-1 /(p-1)}$

In [6, 7], Galaktionov, Mochizuki and Suzuki, had also revealed that the critical case $p=m+(2 / N)$ belongs to the blow-up case, see also $[8,9]$.

In 2010, Payne et al. [10] considered a semilinear heat equation with nonlinear boundary condition $(m=1$ in (1)) and established conditions on nonlinearities sufficient to guarantee that $u(x, t)$ exists for all time $t>0$ as well as conditions on data forcing the solution $u(x, t)$ to blow up at some finite time $t^{*}$. When $N=1$, the blow-up phenomena for the solutions of the porous medium equation with nonlinear flux on the boundary had also been studied by several authors $[11,12]$. For other interesting results on the large time behavior on the solutions of the porous medium equation, we refer the reader to papers [13-16].

Inspired by the above papers, we will study the blow-up phenomena for the solutions of the porous medium equation with nonlinear flux on the boundary in higher dimensional space $(N \geq 2)$. In fact, we find that if the absorption is more 
powerful than the boundary flux, then the solutions of the problem (1)-(3) exist for all time on a bounded star-shaped region. On the other hand, if the boundary flux is more powerful, then the solutions of the problem (1)-(3) blowup at a finite time. Moreover, we will give the upper-bound estimates for the blow-up time.

The paper is organized as follows. In Section 2, we concentrate our attention on the conditions of the global existence for the solutions of the problem (1)-(3). Section 3 is devoted to the investigation of the blow-up phenomena for the solutions of the problem (1)-(3).

\section{Criterion for Global Existence}

In this section, we investigate the global solutions of problem (1)-(3). The main result of this section is the following theorem.

Theorem 1. Let $\Omega$ be a bounded star-shaped region and assume that $q>m$ satisfy

$$
2 q<m+p
$$

If $f$ and $g$ satisfy the following conditions:

$$
\begin{gathered}
f(\xi) \geq k_{1} \xi^{p}, \quad \xi \geq 0, \\
0 \leq g(\xi) \leq k_{2} \xi^{q}, \quad \xi \geq 0,
\end{gathered}
$$

where $k_{1}, k_{2}$ are nonnegative constants, then the nonnegative solutions $u(x, t)$ of the problem (1)-(3) do not blow up.

Proof. Let

$$
\Phi(t)=\int_{\Omega} u^{2} d x
$$

Differentiating (7) and making use of (1), we obtain that

$$
\Phi^{\prime}(t)=2 \int_{\Omega} u u_{t} d x=2 \int_{\Omega} u\left[\Delta u^{m}-f(u)\right] d x .
$$

From the hypothesis (5), we get

$$
\Phi^{\prime}(t) \leq 2 \int_{\Omega} u\left(\Delta u^{m}-k_{1} u^{p}\right) d x .
$$

By (2), (6) and the divergence theorem, we have

$$
\begin{aligned}
\int_{\Omega} u \Delta u^{m} d x & =\int_{\partial \Omega} u \nabla u^{m} \cdot v d s-\int_{\Omega} \nabla u \cdot \nabla u^{m} d x \\
& =\int_{\partial \Omega} u \frac{\partial u^{m}}{\partial v} d s-\int_{\Omega} \nabla u \cdot \nabla u^{m} d x \\
& \leq k_{2} \int_{\partial \Omega} u^{q+1} d s-\int_{\Omega} \nabla u \cdot \nabla u^{m} d x .
\end{aligned}
$$

Here we used the identity $\operatorname{div}\left(u \nabla u^{m}\right)=u \Delta u^{m}+\nabla u \cdot \nabla u^{m}$. By the divergence theorem again, we get

$$
\int_{\partial \Omega}\left(u^{q+1} x\right) \cdot v d s=\int_{\Omega} \operatorname{div}\left(u^{q+1} x\right) d x
$$

Let

$$
\rho_{0}=\min _{\partial \Omega}(x \cdot \nu), \quad d=\max _{\partial \Omega}|x|
$$

Point out that $\rho_{0}$ is positive because $\Omega$ is star-shaped by hypothesis. Notice also that

$$
\begin{aligned}
\operatorname{div}\left(u^{q+1} x\right) & =\operatorname{div}\left[\left(u^{(m+1) / 2}\right)^{2(q+1) /(m+1)} x\right] \\
& =N u^{q+1}+\frac{2(q+1)}{m+1} u^{(2 q-m+1) / 2}\left(x \cdot \nabla u^{(m+1) / 2}\right) .
\end{aligned}
$$

We thus have

$$
\begin{aligned}
\int_{\partial \Omega} u^{q+1} d s \leq & \frac{N}{\rho_{0}} \int_{\Omega} u^{q+1} d x+\frac{2 d(q+1)}{\rho_{0}(m+1)} \\
& \times \int_{\Omega} u^{(2 q-m+1) / 2}\left|\nabla u^{(m+1) / 2}\right| d x .
\end{aligned}
$$

On the another hand

$$
\begin{aligned}
\nabla u \cdot \nabla u^{m} & =\nabla u \cdot\left(m u^{m-1} \nabla u\right) \\
& =m\left(u^{(m-1) / 2} \nabla u\right)\left(u^{(m-1) / 2} \nabla u\right) \\
& =\frac{4 m}{(m+1)^{2}}\left|\nabla u^{(m+1) / 2}\right|^{2} .
\end{aligned}
$$

Therefore, from (10)-(15), we have

$$
\begin{aligned}
\Phi^{\prime}(t) \leq & 2 k_{2} \int_{\partial \Omega} u^{q+1} d s-2 \int_{\Omega} \nabla u \cdot \nabla u^{m} d x \\
& -2 k_{1} \int_{\Omega} u^{p+1} d x \\
\leq & \frac{2 k_{2} N}{\rho_{0}} \int_{\Omega} u^{q+1} d x \\
& +\frac{4 k_{2} d(q+1)}{\rho_{0}(m+1)} \int_{\Omega} u^{(2 q-m+1) / 2}\left|\nabla u^{(m+1) / 2}\right| d x \\
& -\frac{8 m}{(m+1)^{2}} \int_{\Omega}\left|\nabla u^{(m+1) / 2}\right|^{2} d x-2 k_{1} \int_{\Omega} u^{p+1} d x .
\end{aligned}
$$

We obtain from the Young inequality that

$$
\begin{aligned}
& \int_{\Omega} u^{(2 q-m+1) / 2}\left|\nabla u^{(m+1) / 2}\right| d x \\
& \leq \frac{\sigma}{2} \int_{\Omega} u^{2 q-m+1} d x+\frac{1}{2 \sigma} \int_{\Omega}\left|\nabla u^{(m+1) / 2}\right|^{2} d x,
\end{aligned}
$$

where

$$
\sigma=\frac{k_{2} d(q+1)(m+1)}{4 m \rho_{0}}
$$


This $\sigma$ leads to

$$
\begin{gathered}
\frac{4 k_{2} d(q+1)}{\rho_{0}(m+1)} \int_{\Omega} u^{(2 q-m+1) / 2}\left|\nabla u^{(m+1) / 2}\right| d x \\
\leq \frac{8 m}{(m+1)^{2}} \sigma^{2} \int_{\Omega} u^{2 q-m+1} d x \\
+\frac{8 m}{(m+1)^{2}} \int_{\Omega}\left|\nabla u^{(m+1) / 2}\right|^{2} d x .
\end{gathered}
$$

Combining this with (16), we get

$$
\begin{aligned}
\Phi^{\prime}(t) \leq & \frac{2 k_{2} N}{\rho_{0}} \int_{\Omega} u^{q+1} d x+\frac{8 m}{(m+1)^{2}} \sigma^{2} \\
& \times \int_{\Omega} u^{2 q-m+1} d x-2 k_{1} \int_{\Omega} u^{p+1} d x .
\end{aligned}
$$

Let

$$
\alpha=\frac{q-m}{p-q}
$$

Therefore, the hypotheses that $q>m$ and $2 q<m+p$ imply that

$$
0<\alpha<1
$$

So, by Hölder's inequality, we have

$$
\int_{\Omega} u^{2 q-m+1} d x \leq\left(\int_{\Omega} u^{q+1} d x\right)^{\alpha}\left(\int_{\Omega} u^{p+1} d x\right)^{1-\alpha}
$$

For $\epsilon>0$, we obtain from (23) that

$$
\begin{aligned}
\int_{\Omega} u^{2 q-m+1} d x & \leq\left(\epsilon \int_{\Omega} u^{p+1} d x\right)^{1-\alpha}\left(\epsilon^{(\alpha-1) / \alpha} \int_{\Omega} u^{q+1} d x\right)^{\alpha} \\
& \leq(1-\alpha) \epsilon \int_{\Omega} u^{p+1} d x+\alpha \epsilon^{(\alpha-1) / \alpha} \int_{\Omega} u^{q+1} d x .
\end{aligned}
$$

Thus, inserting (24) in (20), we obtain

$$
\begin{aligned}
\Phi^{\prime}(t) \leq & \frac{2 k_{2} N}{\rho_{0}} \int_{\Omega} u^{q+1} d x+\frac{8 m}{(m+1)^{2}} \sigma^{2} \\
& \times\left\{(1-\alpha) \epsilon \int_{\Omega} u^{p+1} d x+\alpha \epsilon^{(\alpha-1) / \alpha} \int_{\Omega} u^{q+1} d x\right\} \\
& -2 k_{1} \int_{\Omega} u^{p+1} d x \\
= & \left(\frac{2 k_{2 N}}{\rho_{0}}+\frac{8 m \sigma^{2} \alpha}{(m+1)^{2}} \epsilon^{(\alpha-1) / \alpha}\right) \\
& \times \int_{\Omega} u^{q+1} d x+\left(\frac{8 m \sigma^{2} \epsilon(1-\alpha)}{(m+1)^{2}}-2 k_{1}\right) \\
& \times \int_{\Omega} u^{p+1} d x=M_{1} \int_{\Omega} u^{q+1} d x-M_{2} \int_{\Omega} u^{p+1} d x
\end{aligned}
$$

where

$$
\begin{gathered}
M_{1}=\frac{2 k_{2 N}}{\rho_{0}}+\frac{8 m \sigma^{2} \alpha}{(m+1)^{2}} \epsilon^{(\alpha-1) / \alpha}>0, \\
M_{2}=2 k_{1}-\frac{8 m \sigma^{2} \epsilon(1-\alpha)}{(m+1)^{2}},
\end{gathered}
$$

and let $\epsilon$ be sufficiently small to ensure $M_{2}>0$. By Hölder's inequality again, we have

$$
\int_{\Omega} u^{q+1} d x \leq\left(\int_{\Omega} u^{p+1} d x\right)^{(q+1) /(p+1)}|\Omega|^{(p-q) /(p+1)},
$$

where we assume throughout the paper that $|\Omega|=\int_{\Omega} d x$ is the measure of $\Omega$. Using (25) and (27), we obtain

$$
\begin{aligned}
\Phi^{\prime}(t) \leq & M_{1}\left(\int_{\Omega} u^{p+1} d x\right)^{(q+1) /(p+1)} \\
& \times\left\{|\Omega|^{(p-q) /(p+1)}-\frac{M_{2}}{M_{1}}\left(\int_{\Omega} u^{p+1} d x\right)^{(p-q) /(p+1)}\right\} .
\end{aligned}
$$

Moreover, using Hölder's inequality once more, we have

$$
\Phi(t)=\int_{\Omega} u^{2} d x \leq\left(\int_{\Omega} u^{p+1} d x\right)^{2 /(p+1)}|\Omega|^{(p-1) /(p+1)},
$$

that is,

$$
\left(\int_{\Omega} u^{p+1} d x\right)^{(p-q) /(p+1)} \geq \Phi(t)^{(p-q) / 2}|\Omega|^{(1-p)(p-q) / 2(p+1)} .
$$

Finally, from (28) and (30), we obtain

$$
\begin{aligned}
\Phi^{\prime}(t) \leq & M_{1}\left(\int_{\Omega} u^{p+1} d x\right)^{(q+1) /(p+1)} \\
& \times\left\{|\Omega|^{(p-q) /(p+1)}\right.
\end{aligned}
$$

$$
\left.-\frac{M_{2}}{M_{1}} \Phi(t)^{(p-q) / 2}|\Omega|^{(1-p)(p-q) / 2(p+1)}\right\} .
$$

We deduced from (31) that $\Phi(t) \leq \max \left\{\Phi(0),\left(M_{2} / M_{1}\right)^{2 /(q-p)}\right.$ $|\Omega|\}$. On the other hand, $\Phi(t)$ is nonnegative function by assumption. So that $\Phi(t)$ keeps bounded continuously under the conditions given in Theorem 1 , the solutions exsit for all time $t>0$. That is, we find that the global solution exists when the absorption is more powerful than the nonlinear boundary flux and this accomplishes the proof of Theorem 1.

\section{Criterion for Blow-Up}

In this section, we concentrate on the finite time $t^{*}$ on which blow-up occurs. We construct two auxiliary functions to redefine $f$ and $g$, then the nonlinear boundary-flux is more powerful than the absorption, and we obtain the following result. 
Theorem 2. Suppose

$$
0 \leq \alpha \leq \beta
$$

Let

$$
\begin{gathered}
F(\xi)=\int_{0}^{\xi} f(\eta) d \eta-\frac{m(m-1)}{2} \int_{0}^{\xi}|\nabla \eta|^{2} \eta^{m-2} d \eta, \\
G(\xi)=\int_{0}^{\xi} g(\eta) d \eta, \\
\Psi(t)=2 \int_{\partial \Omega} G(u) d s-\int_{\Omega} \nabla u \cdot \nabla u^{m} d x-2 \int_{\Omega} F(u) d x .
\end{gathered}
$$

If

$$
\begin{gathered}
\Psi(0)>0, \\
\xi f(\xi) \leq 2(1+\alpha) F(\xi), \quad \xi \geq 0, \\
\xi g(\xi) \geq 2(1+\beta) G(\xi), \quad \xi \geq 0,
\end{gathered}
$$

then the solutions $u(x, t)$ of the problem (1)-(3) blow up at time $t^{*}<T$ with

$$
T=\frac{\Phi(0)}{2 \beta(1+\beta) \Psi(0)} .
$$

Here $\Phi(t)$ is defined in (7). Moreover, if $\beta=0$, then $T=\infty$.

Proof. Differentiating (7) and using the hypothesis (33), we have

$$
\begin{aligned}
\Phi^{\prime}(t)= & 2 \int_{\Omega} u u_{t} d x=2 \int_{\Omega} u\left[\Delta u^{m}-f(u)\right] d x \\
= & 2 \int_{\partial \Omega} u g(u) d s-2 \int_{\Omega} \nabla u \cdot \nabla u^{m} d x \\
& -2 \int_{\Omega} u f(u) d x \geq 2(1+\beta) \Psi(t) .
\end{aligned}
$$

Differentiating (33), we thus obtain from (15) that

$$
\begin{aligned}
\Psi^{\prime}(t)= & 2 \int_{\partial \Omega} g(u) u_{t} d s-\frac{4 m}{(m+1)^{2}} \int_{\Omega}\left(\left|\nabla u^{(m+1) / 2}\right|^{2}\right)_{t} d x \\
& -2 \int_{\Omega} f(u) u_{t} d x+m(m-1) \int_{\Omega}|\nabla u|^{2} u^{m-2} u_{t} d x
\end{aligned}
$$

Note the identity that

$$
\begin{aligned}
\left(\left|\nabla u^{(m+1) / 2}\right|^{2}\right)_{t}= & \frac{(m+1)^{2}(m-1)}{4} \\
& \times|\nabla u|^{2} u^{m-2} u_{t}+\frac{(m+1)^{2}}{2} u^{m-1} \nabla u \cdot \nabla u_{t} .
\end{aligned}
$$

So, from (37), we get

$$
\begin{aligned}
\Psi^{\prime}(t)= & 2 \int_{\partial \Omega} g(u) u_{t} d s-2 \int_{\Omega} \nabla u_{t} \cdot \nabla u^{m} d x \\
& -2 \int_{\Omega} f(u) u_{t} d x .
\end{aligned}
$$

Therefore,

$$
\Psi^{\prime}(t)=2 \int_{\Omega} u_{t}^{2} d x>0
$$

Here, we have used the identities

$$
\begin{gathered}
\operatorname{div}\left(u_{t} \nabla u^{m}\right)=u_{t} \Delta u^{m}+\nabla u_{t} \cdot \nabla u^{m} \\
\int_{\Omega} \nabla u_{t} \cdot \nabla u^{m} d x=\int_{\partial \Omega} u_{t} \nabla u^{m} \cdot v d s-\int_{\Omega} u_{t} \Delta u^{m} d x .
\end{gathered}
$$

So, the hypothesis $\Psi(0)>0$ implies that for all $t \in\left(0, t^{*}\right)$, the following inequality holds $(t)>0$ :

$$
\Psi(t)>0 .
$$

By the Schwarz inequality, we have

$$
\left(\Phi^{\prime}(t)\right)^{2}=4\left(\int_{\Omega} u u_{t} d x\right)^{2} \leq 2 \Phi(t) \Psi^{\prime}(t)
$$

Together with (36), we have

$$
\Phi(t) \Psi^{\prime}(t) \geq \frac{1}{2}\left[\Phi^{\prime}(t)\right]^{2} \geq(1+\beta) \Phi^{\prime}(t) \Psi(t) .
$$

That is,

$$
\left(\Psi \Phi^{-(1+\beta)}\right)^{\prime} \geq 0
$$

Integrating this from 0 to $t$, we obtain

$$
\Psi(t)(\Phi(t))^{-(1+\beta)} \geq \Psi(0)(\Phi(0))^{-(1+\beta)}=M .
$$

Substituting (46) in (36) we obtain the differential inequality

$$
\Phi^{\prime}(t) \geq 2(1+\beta) \Psi \geq 2(1+\beta) M \Phi^{1+\beta} .
$$

If $\beta>0$, then

$$
(\Phi(t))^{-\beta} \leq(\Phi(0))^{-\beta}-2 \beta(1+\beta) M t .
$$

This leads to

$$
t^{*} \leq T=\frac{1}{2 \beta(1+\beta) M}(\Phi(0))^{-\beta}=\frac{\Phi(0)}{2 \beta(1+\beta) \Psi(0)} .
$$

If $\alpha=\beta=0$, then

$$
\Phi(t) \geq \Phi(0) e^{2 M t}
$$

holds for $t>0$. This implies that $t^{*}=\infty$ and completes the proof of Theorem 2 .

\section{Acknowledgments}

This paper is supported by the National Natural Science Foundation of China, the Specialized Research Fund for the Doctoral Program of Higher Education of China, the Natural Science Foundation Project of "CQ CSTC" (cstc2012jjA00013), and Scientific and Technological Research Program of Chongqing Municipal Education Commission. 


\section{References}

[1] H. Fujita, "On the blowing up of solutions of the Cauchy problem for $u_{t}=\Delta u+u^{1+\alpha}$," Journal of the Faculty of Science, vol. 13, pp. 109-124, 1966.

[2] K. Hayakawa, "On nonexistence of global solutions of some semilinear parabolic differential equations," Proceedings of the Japan Academy, vol. 49, pp. 503-505, 1973.

[3] K. Kobayashi, T. Sirao, and H. Tanaka, "On the growing up problem for semilinear heat equations," Journal of the Mathematical Society of Japan, vol. 29, no. 3, pp. 407-424, 1977.

[4] D. G. Aronson and H. F. Weinberger, "Multidimensional nonlinear diffusion arising in population genetics," Advances in Mathematics, vol. 30, no. 1, pp. 33-76, 1978.

[5] V. A. Galaktionov, S. P. Kurdjumov, A. P. Mihaulov, and A. A. Samarski1̌, "On unbounded solutions of the Cauchy problem for the parabolic equation $u_{t}=\nabla\left(u^{\sigma} \nabla u\right)+u^{\beta}$," Doklady Akademii Nauk SSSR, vol. 252, no. 6, pp. 1362-1364, 1980.

[6] V. A. Galaktionov, "Blow-up for quasilinear heat equations with critical Fujita's exponents," Proceedings of the Royal Society of Edinburgh A, vol. 124, no. 3, pp. 517-525, 1994.

[7] K. Mochizuki and R. Suzuki, "Critical exponent and critical blow-up for quasilinear parabolic equations," Israel Journal of Mathematics, vol. 98, pp. 141-156, 1997.

[8] T. Kawanago, "Existence and behaviour of solutions for $u_{t}=$ $\Delta\left(u^{m}\right)+u^{l, "}$ Advances in Mathematical Sciences and Applications, vol. 7, no. 1, pp. 367-400, 1997.

[9] H. A. Levine, "The role of critical exponents in blowup theorems," SIAM Review, vol. 32, no. 2, pp. 262-288, 1990.

[10] L. E. Payne, G. A. Philippin, and S. Vernier Piro, "Blowup phenomena for a semilinear heat equation with nonlinear boundary conditon, I," Zeitschrift für Angewandte Mathematik und Physik, vol. 61, no. 6, pp. 999-1007, 2010.

[11] Z. Jiang, S. Zheng, and X. Song, "Blow-up analysis for a nonlinear diffusion equation with nonlinear boundary conditions," Applied Mathematics Letters, vol. 17, no. 2, pp. 193-199, 2004.

[12] F. Li and J. Li, "Global existence and blow-up phenomena for nonlinear divergence form parabolic equations with inhomogeneous Neumann boundary conditions," Journal of Mathematical Analysis and Applications, vol. 385, no. 2, pp. 1005-1014, 2012.

[13] L. Wang, J. Yin, and C. Jin, “ $\omega$-limit sets for porous medium equation with initial data in some weighted spaces," Discrete and Continuous Dynamical Systems B, vol. 18, no. 1, pp. 223-236, 2013.

[14] S. Kamin and L. A. Peletier, "Large time behaviour of solutions of the porous media equation with absorption," Israel Journal of Mathematics, vol. 55, no. 2, pp. 129-146, 1986.

[15] L. Wang and J. Yin, "Complicated asymptotic behavior of solutions for heat equation in some weighted space," Abstract and Applied Analysis, vol. 2012, Article ID 463082, 15 pages, 2012.

[16] J. L. Vázquez, The Porous Medium Equation: Mathematical Theory, Oxford Mathematical Monographs, The Clarendon Press, Oxford, UK, 2008. 


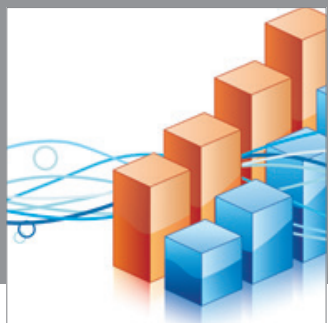

Advances in

Operations Research

mansans

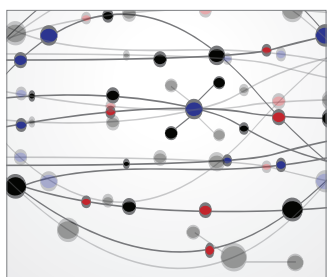

The Scientific World Journal
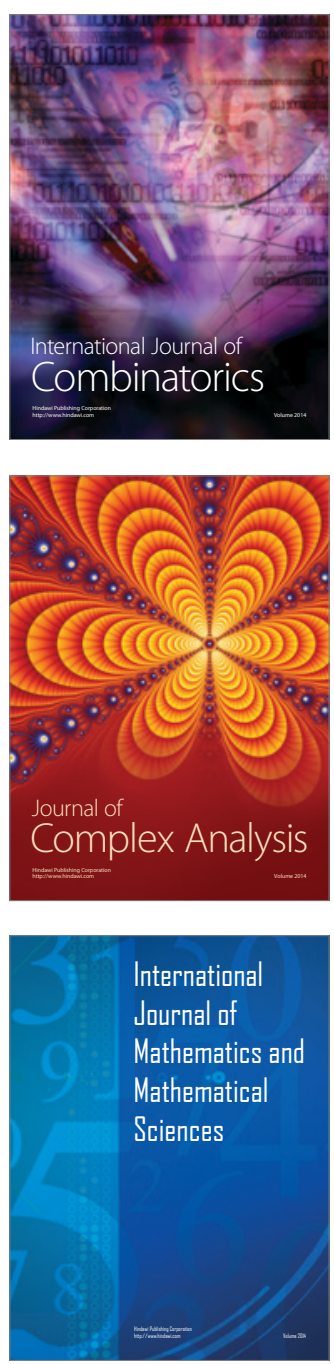
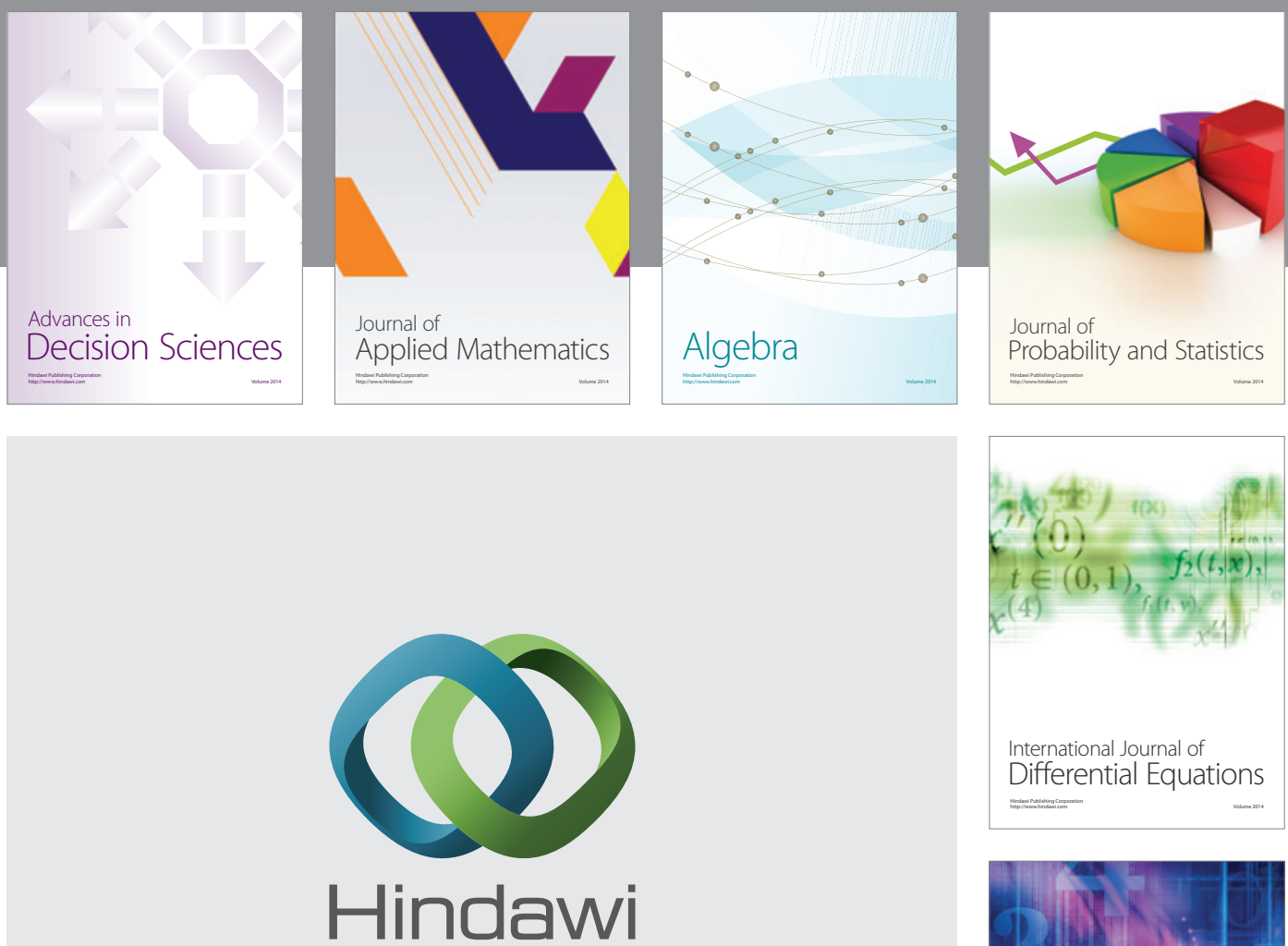

Submit your manuscripts at http://www.hindawi.com
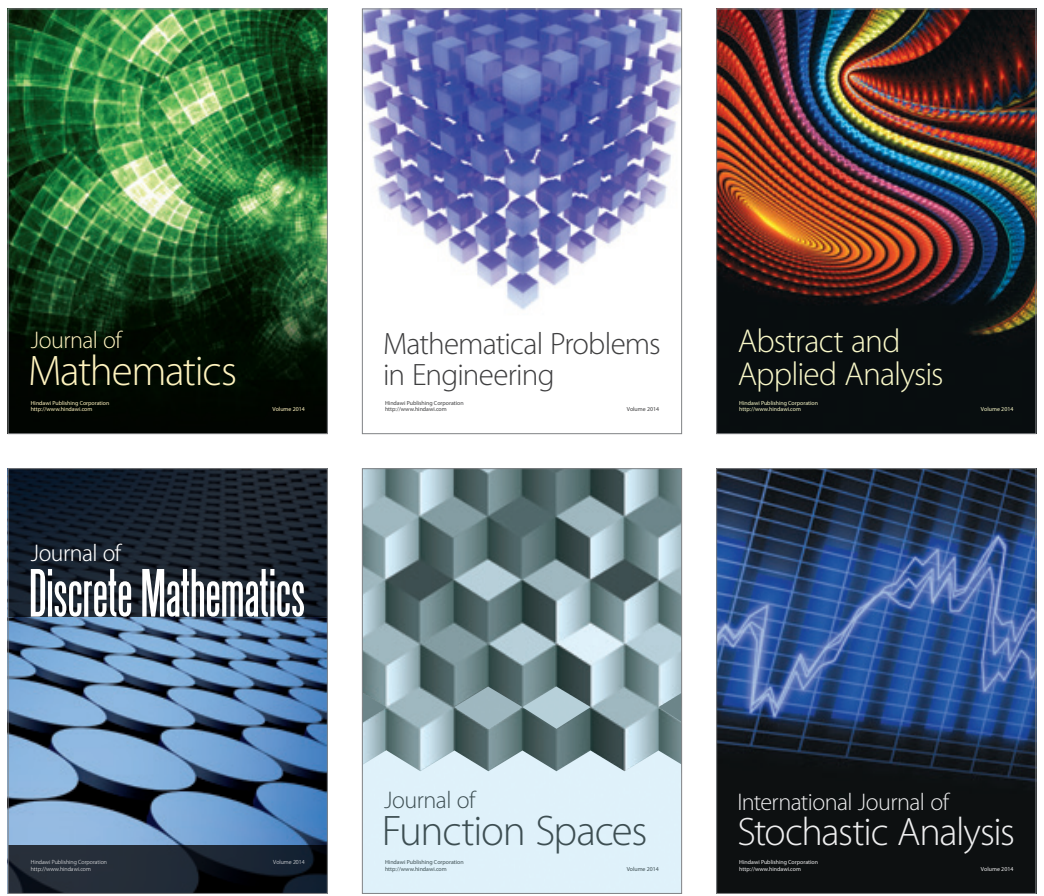

Journal of

Function Spaces

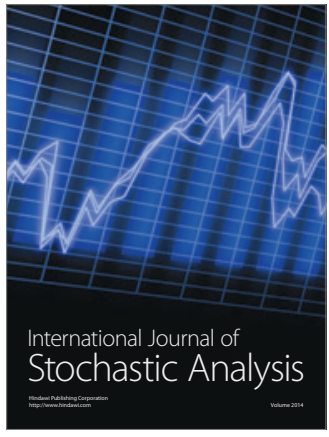

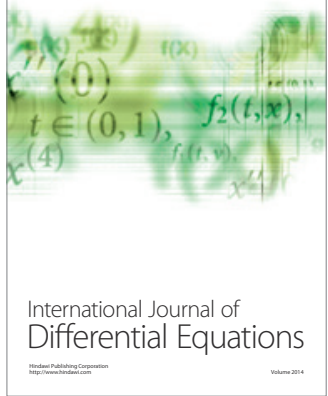
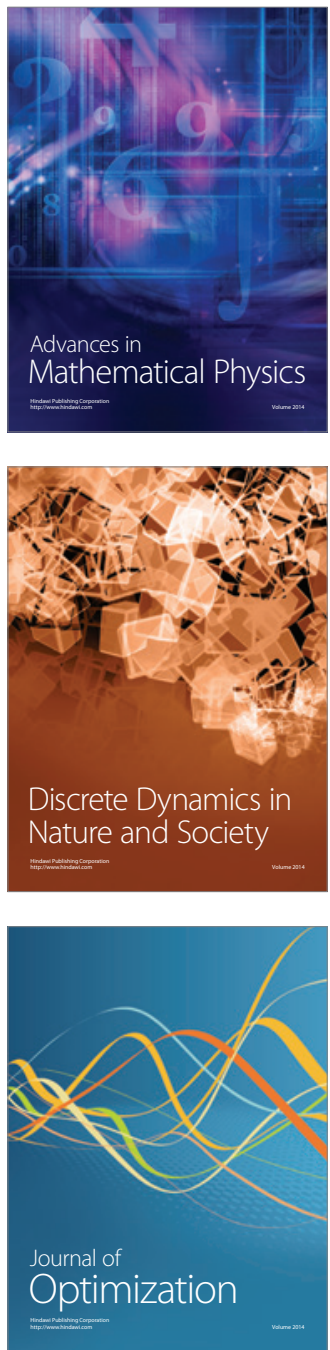